\title{
Comparison of a single-dose vectored thermal pulsation procedure with a 3-month course of daily oral doxycycline for moderate-to-severe meibomian gland dysfunction
}

This article was published in the following Dove Press journal:

Clinical Ophthalmology

\author{
Kerry B Hagen' \\ Raman Bedi ${ }^{2}$ \\ Caroline A Blackie ${ }^{3}$ \\ Kellie J Christenson-Akagi
}

'EyeHealth Northwest, Portland, OR, USA; ${ }^{2}$ ris Advanced Eye Centre, Chandigarh, India; ${ }^{3}$ TearScience, Inc., Morrisville, NC, USA
Correspondence: Caroline A Blackie TearScience, Inc., 5I5I McCrimmon Parkway, Suite 250, Morrisville, NC 27560, USA

Email cblackie@its.jnj.com
Purpose: The aim of this study was to compare the efficacy of a single bilateral 12-minute vectored thermal pulsation (VTP) procedure versus daily oral doxycycline for 3 months for moderate-to-severe meibomian gland dysfunction (MGD).

Methods: This prospective, randomized, parallel-group, single-masked study included 28 subjects who received either a single-dose VTP or 3 months of doxycycline treatment. At baseline and 3 months post treatment, all subjects were evaluated for the following: dry eye symptoms with a standard dry eye questionnaire (the Standard Patient Evaluation for Eye Dryness [SPEED]), meibomian gland (MG) function by counting the number of glands yielding liquid secretion with the MG evaluator (MGE), tear breakup time (TBUT) and corneal and conjunctival staining.

Results: In the VTP group, at 3 months, there was a significant improvement in MG function $(4.00 \pm 1.47$ to $7.73 \pm 5.53)$, SPEED score (11.00 \pm 3.30 to $5.42 \pm 2.15)$, TBUT $(6.26 \pm 2.01$ to $8.44 \pm 1.81)$, corneal staining $(0.38 \pm 0.50$ to $0.12 \pm 0.33)$ and conjunctival staining $(1.69 \pm 1.93$ to $0.62 \pm 0.85)$. In the doxycycline group, there was a significant improvement in MG function (4.63 \pm 1.41 to $10.63 \pm 5.91)$, SPEED score (13.42 \pm 4.17 to $9.42 \pm 5.47)$ and conjunctival staining $(2.38 \pm 1.88$ to $1.13 \pm 1.51)$, but the improvement in TBUT $(6.90 \pm 2.56$ to $7.59 \pm 2.03)$ and corneal staining ( $0.21 \pm 0.41$ to $0.13 \pm 0.34$ ) was not statistically significant ( $p=0.262$ and $p=0.414$, respectively). At 3 months, SPEED score was significantly better in the VTP group $(p<0.05)$; other parameters were comparable between the two groups.

Conclusion: A single 12-minute bilateral VTP procedure was significantly more effective than the 3-month daily course of oral doxycycline at improving the dry eye symptoms secondary to MGD. A single 12-minute VTP treatment was at least as effective as a dose of doxycycline for 3 months, in improving MG function and all measured signs of MGD. Given the minimal risk profile of the single VTP procedure over long-term doxycycline use, a single VTP presents a favorable alternative to long-term antibiotic use.

Keywords: meibomian gland dysfunction, dry eye, vectored thermal pulsation, LipiFlow

\section{Introduction}

Meibomian gland dysfunction (MGD) has recently been recognized as the leading cause of dry eye. ${ }^{1}$ MGD is a highly prevalent, chronic and progressive condition that can be diagnosed by observable compromise to the function and/or structure of the meibomian glands (MGs). Later stage disease can present with additional findings including ocular surface inflammation, changes to the tear film and lid margin and dry eye symptoms. ${ }^{1,2}$ 
While there are many adjunctive treatments available for the ongoing management of MGD, eg, daily heat and massage of the external lid surface, successful long-term rehabilitation of MG function usually requires that gland obstruction be treated by evacuating the gland contents. ${ }^{3}$ Vectored thermal pulsation (VTP), described elsewhere, ${ }^{4}$ is specifically designed and has been shown to be highly effective at evacuating the contents of the MGs of all four eyelids simultaneously in a single 12 -minute treatment. ${ }^{3}$ The successful use of VTP has been shown in multiple patient cohorts with MGD, including those with dry eye, refractory dry eye, refractory dry eye post LASIK, Sjögren's disease and contact lens wearers..$^{5-8}$ Previous clinical studies have shown sustained improvement in MG function and dry eye symptoms with a single 12-minute VTP procedure over 12-36 months. ${ }^{5,9}$

Despite the awareness that obstruction is the core mechanism in almost all MGD, the field lacks consensus on generalized treatment recommendations for the evacuation of gland contents for MGD. In the cases of more advanced MG disease, inflammation of the eyelid tissue may also require treatment. Similarly, there is little consensus as to how to manage inflammation of the eyelid tissue in laterstage disease. ${ }^{10}$ Antibiotic agents, including macrolides, and tetracycline and its derivatives have been shown to be beneficial for the management of various chronic inflammatory conditions, ${ }^{11}$ and doxycycline is a long-acting analog of tetracycline that has been effectively used for the treatment of dry eye disease. ${ }^{12-16}$ Its therapeutic efficacy has been attributed to various factors including its ability to inhibit the synthesis or activation of proinflammatory mediators in the corneal epithelium and its antimicrobial and antichemotactic properties, which leads to improvement in dry eye symptoms and tear film stability. ${ }^{12,14}$ However, long-term use of doxycycline can be problematic due to the risk of side effects. ${ }^{14,15}$

Analysis of biochemical properties of meibomian secretions following oral doxycycline treatment has shown improvement in the composition and characteristics of the MG secretions (MGS). ${ }^{12}$ These changes in the properties of meibum appear to improve its fluidity and enhance gland function. The purpose of the current study was to compare the effect of a 3-month course of daily oral doxycycline therapy and a single 12-minute VTP procedure on moderateto-severe MGD.

\section{Methods}

This prospective, randomized, parallel-group, single-masked study was conducted in compliance with the tenets of
Declaration of Helsinki. The study was approved by Sterling Institutional Review Board (Atlanta, GA, USA). All study participants were fully consented, and written informed consent was obtained.

\section{Study design}

This study included 28 subjects with moderate-to-severe MGD, who were randomized to receive either doxycycline treatment or VTP procedure in 1:1 ratio (14 patients in the doxycycline group and 14 in the VTP group). The subjects randomized to the doxycycline group were administered daily oral doxycycline for 3 months (100 mg twice daily [BID] for the first 14 days and $100 \mathrm{mg}$ once daily for days 15-90) and those randomized to the VTP group received a single bilateral 12-minute VTP procedure with LipiFlow System (TearScience, Inc., Morrisville, NC, USA). The single examiner $(\mathrm{KBH})$ was masked to the treatment type assigned to each subject. To ensure masking of the examiner, subjects randomized to VTP procedure were treated by another practitioner and subjects were instructed to not comment on their treatment type to the examiner at the final examination.

\section{Inclusion criteria}

Subjects eligible for participating in the study met the following inclusion criteria: aged 21 years or older; provided written informed consent and were willing to comply with the study procedures and follow-up schedule; a Standard Patient Evaluation for Eye Dryness (SPEED) score of $\geq 6$ at the baseline visit, ${ }^{17}$ evidence of MGD based on the number of functional MGs $\leq 6$ (meibomian glands yielding liquid secretion [MGYLS] using the MG evaluator [MGE; TearScience, Inc.]); ${ }^{18}$ no previous history of allergy or sensitivity to doxycycline; no use of additional anti-inflammatory agents for the prior 3 months. To ensure equivalence between the two groups regarding the likelihood of improvement, both lower eyelids of all subjects were required to have $\geq 50 \%$ observable MG structure determined by retroillumination meiboscopy.

\section{Exclusion criteria}

Subjects were excluded if there was any evidence of coexisting ocular conditions that may potentially pose an increased risk of procedure-related injury, limit the effectiveness of treatment or interfere with its assessment. These ocular conditions included active ocular inflammation in either eye, Demodex blepharitis, ocular surgery within the past 3 months of baseline examination, or structural ocular 
surface and eyelid abnormalities. In addition, patients with Sjögren's syndrome, rheumatoid arthritis, systemic disease resulting in dry eye, known autoimmune disease, doxycycline allergy or sensitivity, history of antibiotic therapy at any time within 2 weeks of the commencement of study and those using significant calcium supplementation or other medications that could interfere with doxycycline absorption were also excluded.

\section{Adjunctive treatment}

Subjects were requested to continue using any previously used adjunctive therapy in the same manner as long as they already had been using it for a minimum of 3 months (for instance, artificial tears and/or fish oil supplements, lid scrubs and warm compresses). Starting a new adjunctive therapy for MGD during the study period was not permitted.

\section{Study end points}

The primary end points of the study were SPEED and MG function (MGYLS) scores. Additional study parameters included tear breakup time (TBUT), corneal and conjunctival staining and slit-lamp examination. All subjects were evaluated at baseline and 3 months post treatment.

The SPEED questionnaire evaluated the frequency and severity of dry eye symptoms over the past 3 months. The total SPEED score was calculated as the sum of the frequency and severity scores for all symptoms over a range from 0 to 28 .

The MG assessment was performed at a slit lamp using a hand-held instrument, the MGE (TearScience, Inc.), to ensure consistency of pressure applied to the glands. ${ }^{4,19}$ The MGE approximates the force of a deliberate blink in yielding secretions from the glands. ${ }^{18}$ This instrument was held in place for 10-15 seconds, while the number of MGs expressing clear liquid secretions along the entire lower eyelid was counted and recorded.

TBUT was performed with Dry Eye Test (DET) fluorescein strips (Amcon Laboratories, St Louis, MO, USA) using previously described DET method. ${ }^{20}$ The DET strip was moistened with a single drop of saline and held vertically to allow the excess saline to run off. Patients were instructed to look down and toward the hand opposite to the eye receiving the stain. After retracting the upper eyelid, the strip was applied to superior temporal bulbar conjunctiva. To observe fluorescein breakup, the standard slit-lamp cobalt blue filter was used. Tear film breakup was defined as the first observed breakup of the tear film following the third blink. The measurements were recorded with the help of a stop watch, and an average of three readings was obtained for data analysis.

For corneal and conjunctival staining, the respective dyes were instilled using commercially available saline and standard fluorescein (Akorn, Inc., Farmington, CT, USA) and lissamine green strips (Bernell Corporation, Mishawaka, IN, USA). Each strip was moistened with a single drop of saline. The moistened strips were used to place the stain in the inferior cul-de-sac of each eye. After waiting for 90 seconds post dye instillation, the ocular surface (cornea and conjunctiva) was evaluated for staining.

Based on the report of the National Eye Institute/Industry (NEI) Workshop on Clinical Trials in Dry Eye, ${ }^{21}$ corneal fluorescein (CF) staining was graded in five regions of the cornea on a scale of 0 (none) to 3 (severe) with a total score of 15. Conjunctival lissamine green (CLG) staining was graded on a scale of 0 (none) to 3 (severe) over six regions of the conjunctiva, with scores ranging from 0 to 18 .

\section{LipiFlow procedure}

In the subjects randomized to receive the LipiFlow, the procedures were administered in accordance with the device labeling. The LipiFlow, designed to heat and simultaneously evacuate the MG contents, has been extensively described elsewhere. ${ }^{4}$ In brief, one to two drops of topical anesthesia were applied prior to the bilateral application of the activators. The inner portion of the activator applied a constant temperature of $42.5^{\circ} \mathrm{C}$ to the tarsal conjunctiva of upper and lower eyelids. Simultaneously, the outer portion of the activator applied directional, pulsatile pressure to the external eyelid surfaces (maximum $6 \mathrm{psi}$ ) for the 12-minute treatment cycle. Consequently, the subjects felt warmth and pressure on their eyelids during the 12-minute bilateral procedure. All subjects were monitored throughout the process, and both the eyes were examined at the slit lamp at the end of the procedure.

\section{Statistical analysis}

The study sample size was based on a power analysis of the primary study end points and the null hypothesis that the single VTP treatment would be non-inferior to the 3-month daily dose of doxycycline (power $80 \%$, alpha $=0.05$ ).

Data analysis was performed using SPSS software for Windows version 17.0 (SPSS Inc., Chicago, IL, USA). The data were examined for outliers using outlier labeling method with a multiplier of 2.2, and one outlier was removed from the analysis of SPEED scores of the VTP treatment group. The normality of data was checked using Shapiro-Wilk 
test, and a threshold of $<0.05$ was considered significant. For normally distributed data, independent $t$-test and paired $t$-test were used for comparison between the groups and within the group, respectively; otherwise, their nonparametric counterparts, ie, Mann-Whitney $U$ test and Wilcoxon signedrank test had been used. A $p$-value of $<0.05$ was considered statistically significant.

\section{Results}

Of the 28 subjects recruited in the study, two subjects in the doxycycline group discontinued their treatment regimen due to stomach upset/intolerance to study medication and one in the VTP group was lost to follow up. Therefore, these subjects were removed from the analysis. The mean age of subjects included in the VTP and doxycycline groups was 51.7 \pm 15.6 and $50.4 \pm 14.4$ years, respectively $(p=0.84)$. At baseline, all the study parameters were comparable between the two groups (Table 1).

At 3 months, SPEED scores, MGYLS and TBUT were significantly improved from their baseline levels in the VTP group ( $p<0.005$; Figures $1-3$ ). Similarly, there was a significant improvement in CF and CLG staining in the VTP group ( $p=0.020$ and $p=0.002$, respectively). In the doxycycline group, SPEED scores, MGYLS (Figures 1 and 2) and CLG staining improved significantly from their pretreatment levels $(p<0.005)$, but the improvement in TBUT (Figure 3$)$ and CF staining did not reach statistical significance $(p=0.262$ and $p=0.414$, respectively).

Specifically, at 3 months, the mean SPEED scores

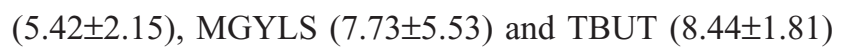
were significantly improved from their baseline levels in the VTP group ( $p<0.005$; Figures $1-3$ ). Similarly, there was a significant improvement in CF and CLG staining in the VTP group $(0.12 \pm 0.33$ and $0.62 \pm 0.83 ; p=0.020$ and $p=0.002$, respectively). In the doxycycline group, SPEED scores

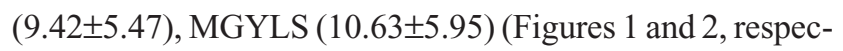
tively) and CLG staining (1.13 \pm 1.51$)$ improved significantly from their pretreatment levels $(p<0.005)$, but the improvement in TBUT (7.59 \pm 2.03 ; Figure 3$)$ and CF staining $(0.13 \pm 0.34)$ did not reach statistical significance $(p=0.262$ and $p=0.414$, respectively).

Comparison between the two groups at 3 months revealed that the mean SPEED scores of the VTP group were significantly better than the doxycycline group $(p=0.03)$ (Figure 1). MGYLS and TBUT were found to be comparable between the two groups ( $p=0.096$ and $p=0.155$, respectively; Figures 2 and 3). Similarly, the two groups were comparable for $\mathrm{CF}$ staining $(0.12 \pm 0.33$ and $0.13 \pm 0.34$ for the VTP and doxycycline groups, respectively; $p=0.918$ ) and CLG staining $(0.62 \pm 0.85$ and $1.13 \pm 1.51$ for the VTP and doxycycline groups, respectively; $p=0.299$ ).

\section{Discussion}

The doxycycline outcomes in the current study were similar to those published previously. ${ }^{12-16}$ Subjective symptom scores (assessed using different scales) have been reported to significantly improve following 3-4 weeks of doxycycline treatment regimens, using doses as high as $400 \mathrm{mg}$ doxycycline per day. ${ }^{14-16}$ In addition, daily administration of $200 \mathrm{mg}$ oral doxycycline (100 mg BID for 1-2 months) has been found to improve meibomian gland secretion scores. ${ }^{12,14}$ In this study, a standardized amount of pressure, approximating a deliberate blink, was applied over the glands as opposed to the more variable "mild" or "firm" pressure as described in other studies evaluating the benefits of treating MGD with doxycycline. The standardized pressure with the MGE used in this study better represents the "functional" status of the MG, ie, the ability of the gland to release liquid during the blinking process. Using the standardized pressure provided by the MGE, these study results corroborate some previous studies showing that doxycycline can improve meibomian gland secretion. ${ }^{12,14}$

Doxycycline treatment has also been reported to significantly improve TBUT in some studies; ${ }^{12,15}$ Kashkouli et al, ${ }^{14}$ in contrast, reported slight decrease in TBUT. The 3-month

Table I Baseline data of the two treatment groups

\begin{tabular}{llllll}
\hline Variables & N (eyes) & $\begin{array}{l}\text { Doxycycline group } \\
\text { (mean } \pm \text { SD) }\end{array}$ & N (eyes) & $\begin{array}{l}\text { LipiFlow group } \\
\text { (mean } \pm \text { SD) }\end{array}$ \\
\hline Age (years) & $12 *$ & $50.4 \pm 14.4$ & $67.58 \pm 21.85$ & $13^{*}$ & $51.7 \pm 15.6$ \\
LLT $(\mu \mathrm{m})$ & 24 & $4.63 \pm 1.41$ & 26 & $73.19 \pm 18.42$ & 0.841 \\
MGYLS & 24 & $6.90 \pm 2.56$ & 26 & $4.00 \pm 1.47$ & 0.330 \\
TBUT (seconds) & 24 & $13.42 \pm 4.17$ & 26 & $6.26 \pm 2.01$ & 0.133 \\
SPEED score & $12 *$ & $0.21 \pm 0.41$ & $12^{*}$ & $11.00 \pm 3.30$ & 0.329 \\
CF staining & 24 & $2.38 \pm 1.88$ & 26 & $0.38 \pm 0.50$ & 0.130 \\
CLG staining & 24 & & 26 & $1.69 \pm 1.93$ & 0.119 \\
\hline
\end{tabular}

Note: *The number of patients.

Abbreviations: CF, corneal fluorescein; CLG, conjunctival lissamine green; LLT, lipid layer thickness; MGYLS, meibomian glands yielding liquid secretion; SPEED, Standard Patient Evaluation for Eye Dryness; TBUT, tear breakup time. 


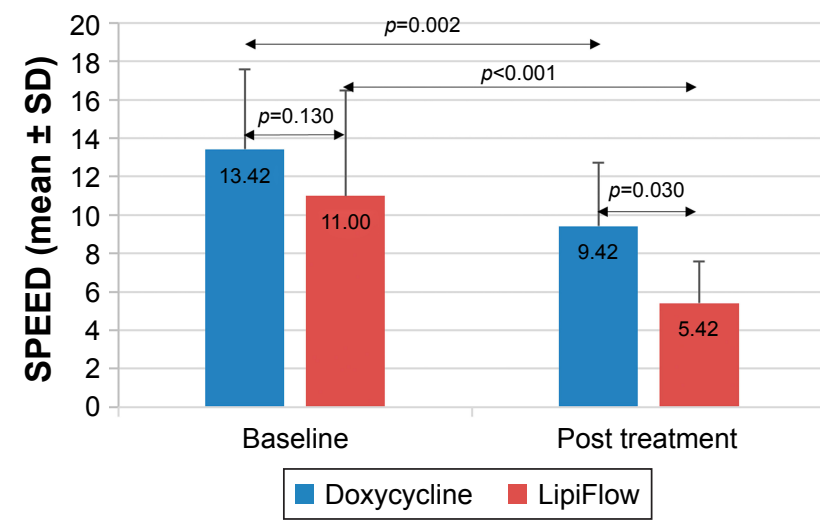

Figure I The mean SPEED scores at baseline and 3 months post treatment for the doxycycline and LipiFlow groups.

Notes: After 3 months, SPEED scores were significantly improved from their baseline values in both the groups $(p<0.005)$. The mean SPEED scores of the LipiFlow group were significantly better than the doxycycline group at 3 months ( $p=0.030$; error bars represent SD).

Abbreviation: SPEED, Standard Patient Evaluation for Eye Dryness.

course of doxycycline in the current study did not show statistically significant improvement in TBUT. To the best of our knowledge, the outcomes of conjunctival staining have not been studied following doxycycline therapy; the mean CLG scores were significantly improved in the current study. Of note, Kashkouli et al ${ }^{14}$ reported the outcomes for ocular surface staining that included the assessment of the entire cornea as well as nasal and temporal interpalpebral conjunctival regions, graded using a modified 4-point categorical scale (grade $0-3$ ). The mean ocular surface staining scores were found to improve significantly following doxycycline therapy (100 mg BID for 1 month). Regarding corneal staining, Zandian et al ${ }^{16}$ reported significant improvement in the mean corneal staining scores with oral doxycycline therapy (100 mg daily for 3 weeks). Similarly, The mean corneal

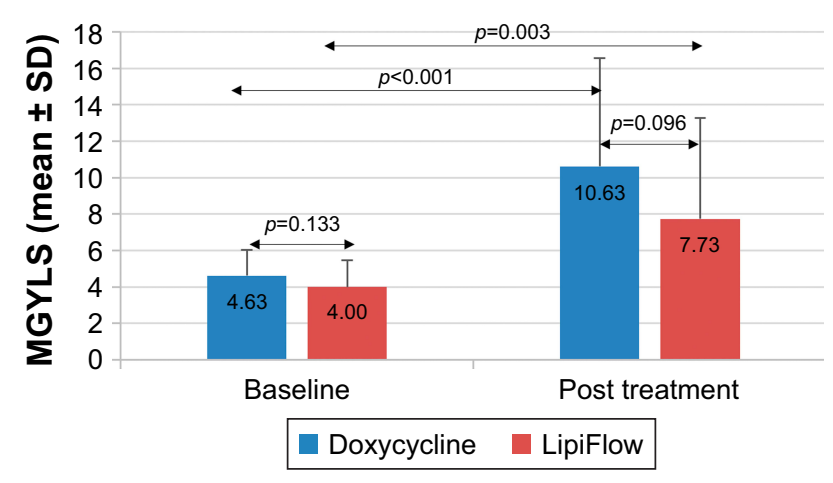

Figure 2 The mean MGYLS scores at baseline and 3 months post treatment for the doxycycline and LipiFlow groups.

Notes: At 3 months post treatment, both the groups demonstrated significant improvement in MGYLS scores from their baseline values $(p<0.005)$. No statistically significant difference in the mean MGYLS scores was observed between the two study groups at 3 months ( $p=0.096$; error bars represent SD).

Abbreviation: MGYLS, meibomian glands yielding liquid secretion.

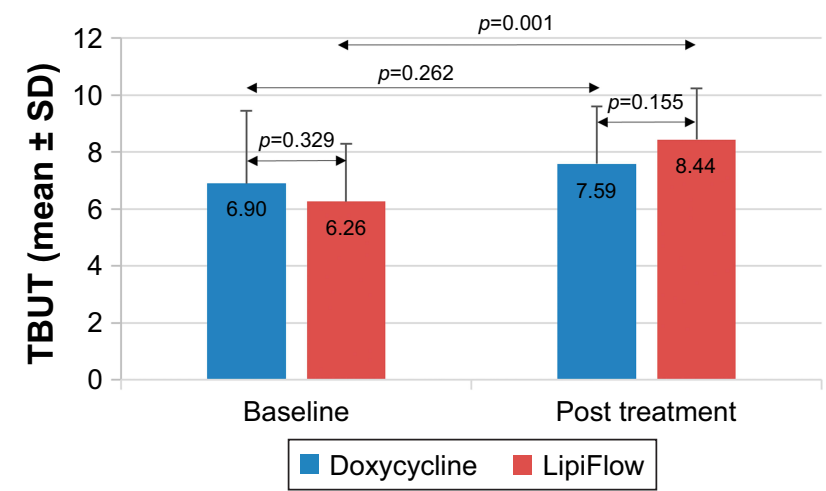

Figure 3 The mean TBUT at baseline and 3 months post treatment for the doxycycline and LipiFlow groups.

Notes: Compared to the baseline, TBUT was significantly improved in the VTP group at 3 months post treatment $(p=0.001)$; however, the improvement was not statistically significant in the doxycycline group $(p=0.262)$. There was no significant difference in the mean TBUT values of the two groups at 3 months $(p=0.155$; error bars represent SD).

Abbreviations: TBUT, tear breakup time; VTP, vectored thermal pulsation.

staining score improvement in the doxycycline group of the current study was not statistically significant. Overall, the differences may be explained, in part, by different grading methods, especially for MGD which has evolved significantly in the past few years, different levels of severity of MGD and dry eye-associated anatomical changes in the ocular surface and eyelid.

The outcomes of a single VTP procedure in the current study are in alignment with the numerous other studies documenting a predictably significant improvement in MG function ${ }^{4,22,23}$ and reduction in dry eye symptoms ${ }^{4,9,19,22,24}$ after a single VTP treatment. While several studies also report significant improvement in TBUT after VTP, ${ }^{4,23}$ there are some that were not able to show statistically significant improvement in TBUT. ${ }^{22,24,25}$ The results of the current study demonstrated significant improvement in TBUT in the VTP-treated eyes. The mean corneal staining scores have been reported to improve significantly following the VTP procedure. ${ }^{4,23}$ Similarly, the mean CF staining scores improved significantly in the VTP group of the current study. The conjunctival staining has not been studied extensively in the previous literature; a single study reported small but statistically significant increase in the mean conjunctival staining scores in the VTP group, at 4 weeks from baseline. ${ }^{4}$ As expected from the overall improvement in ocular surface health in the VTP group of the current study, we found significant improvement in the mean CLG scores.

The mean MGYLS scores of the two groups were found to be not statistically significantly different from one another both pre and post treatment. The VTP procedure is known to improve the MG function, so the study results are in 
alignment with what would be expected. The improvement in gland function as a result of the daily use of doxycycline for 3 months was not as clearly predicted by the literature. The previously reported favorable biochemical changes in MGSs following systemic tetracycline analog administration may explain the improvement in gland function. For example, Foulks et $\mathrm{al}^{12}$ reported changes in the characteristics and biochemical composition of MGSs following 4 weeks of oral doxycycline treatment in patients with MGD. Souchier et $\mathrm{al}^{26}$ reported alterations in the meibomian fatty acid composition after 8-week therapy with minocycline, a tetracycline analog similar to doxycycline. Branched chain fatty acids (BCFAs), particularly isoC20, were found to be decreased. Higher levels of BCFAs are assumed to have detrimental effect on MG epithelium and been correlated with the keratinization of MG. ${ }^{27}$ It has been hypothesized that lower levels of BCFAs and correspondingly higher levels of C16 and C18 saturated fatty acids may enhance the fluidity and stability of the tear film, thus improving the dry eye symptoms. ${ }^{26}$

While this study demonstrates that the daily use of doxycycline for 3 months can improve the signs and symptoms of moderate-to-severe MGD, the use of doxycycline is not without complication. There are many known side effects which serve to decrease patient compliance and may result in the termination of treatment altogether. This was evidenced in the current study; two subjects (14\%) in the doxycycline group discontinued their treatment regimen due to stomach upset/intolerance to study medication. Previous publications reporting on the use of doxycycline for dry eye have documented numerous side effects, such as gastrointestinal problems (esophagitis, nausea, dyspepsia, diarrhea, abdominal cramps and decreased appetite), dermatological effects (itchy skin, urticaria and erythematous papules), hypersensitivity reactions (allergy and fever), increased tendency to bleed and stomatitis. ${ }^{14,15}$ Doxycycline has also been linked to liver failure and teratogenicity. ${ }^{28}$ Depending upon the dose and duration of doxycycline treatment, side effects have been reported in as high as $40 \%$ of the subjects. ${ }^{14,15}$ In addition, medical literature outside ophthalmology has reported some rare side effects such as esophageal ulcers, light-sensitive eruptions, photo-onycholysis and hypersensitivity syndrome reactions, single organ dysfunction and serum sickness such as reaction with doxycycline treatment. ${ }^{29-33}$ Its long-term use has been related to potential development of bacterial resistance and increased risk of secondary infections with other organisms (particularly Candida sp.). ${ }^{34}$ Sub-microbial dosing of doxycycline has been advocated to lessen side effects and risk of microbial resistance, but even at low doses nasopharyngitis, diarrhea, headache, hypertension, aspartate aminotransferase (AST) elevation and abdominal pain have been reported. ${ }^{15,35}$

As a chronic and progressive condition, MGD requires long-term management. The long-term safety concerns with doxycycline use render it a short-term, albeit sometimes necessary, option at best. In contrast, the VTP procedure lasts only 12 minutes, has an exceptional safety profile $\mathrm{e}^{4,22}$ and has been shown to result in sustained clinically significant improvement in gland function and reduced dry eye symptoms observed over 12-36 months. ${ }^{5,9}$ Consistent with the previous findings, no adverse events were found for VTP-treated eyes in this study. A single 12-minute VTP procedure presents a favorable alternative to the long-term use of oral antibiotics.

There are several limitations to this study: 1) it is a small study, thus serving as a pilot study for additional investigations; 2) as is typical in clinical practice, the adjunctive lid hygiene treatments that patients use for MGD and to manage other lid conditions are customized to the needs of the patient. What was consistent between patients was the restriction to make no changes to the at-home therapies for the study period; 3) blinking exercises were not prescribed in this study.

Over its disease course, untreated MGD is considered to result in irreversible anatomical damage to the MGs. Recently, Blackie et $\mathrm{ll}^{5}$ provided clinical evidence in support of the hypothesis that early intervention for MGD optimizes the therapeutic outcomes. They reported significant association between greater mean improvement in MGS scores and patient characteristics such as less severe baseline scores and shorter duration of time between the diagnosis and treatment of MGD. In this study, the subjects had more advanced disease prior to treatment. Even so, the results of a single VTP treatment were compelling. Based on these results, one could hypothesize that VTP in combination with short-term doxycycline use may be a highly effective treatment option for more advanced stages of MGD. The authors hypothesize that such a combined approach may induce positive changes in the fatty acid composition of MGSs which may be better amenable to liquefaction and clearance of obstruction by VTP procedure. It could possibly also improve the duration of treatment effect of a single VTP procedure. Future studies are warranted to evaluate the proposed synergistic role of VTP procedure with short-term doxycycline use.

\section{Conclusion}

In this prospective, randomized, parallel-group, singlemasked study, a single 12-minute bilateral VTP procedure was significantly more effective than the 3-month daily 
course of oral doxycycline at improving the dry eye symptoms secondary to MGD. A single VTP treatment was at least as good as the daily doxycycline in improving $\mathrm{MG}$ function and all measured signs of MGD for moderate-tosevere MGD. Given the minimal risk profile of the single VTP procedure over long-term doxycycline use, a single VTP procedure presents a favorable alternative to long-term antibiotic use.

\section{Disclosure}

$\mathrm{RB}$ is a consultant for TearScience, Inc. CAB is an employee of TearScience, Inc. The other authors report no conflicts of interest in this work.

\section{References}

1. The definition and classification of dry eye disease: report of the Definition and Classification Subcommittee of the International Dry Eye WorkShop (2007). Ocul Surf. 2007;5(2):75-92.

2. Nelson JD, Shimazaki J, Benitez-del-Castillo JM, et al. The international workshop on meibomian gland dysfunction: report of the definition and classification subcommittee. Invest Ophthalmol Vis Sci. 2011;52(4):1930-1937.

3. Blackie CA, Carlson AN, Korb DR. Treatment for meibomian gland dysfunction and dry eye symptoms with a single-dose vectored thermal pulsation: a review. Curr Opin Ophthalmol. 2015;26(4):306-313.

4. Lane SS, DuBiner HB, Epstein RJ, et al. A new system, the LipiFlow, for the treatment of meibomian gland dysfunction. Cornea. 2012; 31(4):396-404.

5. Blackie CA, Coleman CA, Holland EJ. The sustained effect (12 months) of a single-dose vectored thermal pulsation procedure for meibomian gland dysfunction and evaporative dry eye. Clin Ophthalmol. 2016;10:1385-1396.

6. Epitropoulos AT, Goslin K, Bedi R, Blackie CA. Meibomian gland dysfunction patients with novel Sjogren's syndrome biomarkers benefit significantly from a single vectored thermal pulsation procedure: a retrospective analysis. Clin Ophthalmol. 2017;11:701-706.

7. Korb D, Blackie C. A Single Lipiflow treatment increases soft contact lens wearing time and reduces lid wiper epitheliopathy and dry eye symptoms. American Academy of Optometry's 93rd Annual Meeting. Denver: 2014.

8. Schallhorn CS, Schallhorn JM, Hannan S, Schallhorn SC. Effectiveness of an eyelid thermal pulsation procedure to treat recalcitrant dry eye symptoms after laser vision correction. J Refract Surg. 2017;33(1): $30-36$.

9. Greiner JV. Long-term (3 year) effects of a single thermal pulsation system treatment on meibomian gland function and dry eye symptoms. Eye Contact Lens. 2016;42(2):99-107.

10. Qiao J, Yan X. Emerging treatment options for meibomian gland dysfunction. Clin Ophthalmol. 2013;7:1797-1803.

11. Pflugfelder SC, Geerling G, Kinoshita S, et al. Management and therapy of dry eye disease: report of the Management and Therapy Subcommittee of the International Dry Eye WorkShop (2007). Ocul Surf. 2007;5(2):163-178.

12. Foulks GN, Borchman D, Yappert M, Kakar S. Topical azithromycin and oral doxycycline therapy of meibomian gland dysfunction: a comparative clinical and spectroscopic pilot study. Cornea. 2013;32(1): $44-53$.

13. Iovieno A, Lambiase A, Micera A, Stampachiacchiere B, Sgrulletta R, Bonini S. In vivo characterization of doxycycline effects on tear metalloproteinases in patients with chronic blepharitis. Eur J Ophthalmol. 2009;19(5):708-716.
14. Kashkouli MB, Fazel AJ, Kiavash V, Nojomi M, Ghiasian L. Oral azithromycin versus doxycycline in meibomian gland dysfunction: a randomised double-masked open-label clinical trial. Br J Ophthalmol. 2015;99(2):199-204.

15. Yoo SE, Lee DC, Chang MH. The effect of low-dose doxycycline therapy in chronic meibomian gland dysfunction. Korean J Ophthalmol. 2005;19(4):258-263.

16. Zandian M, Rahimian N, Soheilifar S. Comparison of therapeutic effects of topical azithromycin solution and systemic doxycycline on posterior blepharitis. Int J Ophthalmol. 2016;9(7):1016-1019.

17. Ngo W, Situ P, Keir N, Korb D, Blackie C, Simpson T. Psychometric properties and validation of the Standard Patient Evaluation of Eye Dryness questionnaire. Cornea. 2013;32(9):1204-1210.

18. Korb DR, Blackie CA. Meibomian gland diagnostic expressibility: correlation with dry eye symptoms and gland location. Cornea. 2008; 27(10):1142-1147.

19. Finis D, Konig C, Hayajneh J, Borrelli M, Schrader S, Geerling G. Sixmonth effects of a thermodynamic treatment for MGD and implications of meibomian gland atrophy. Cornea. 2014;33(12):1265-1270.

20. Korb DR, Greiner JV, Herman J. Comparison of fluorescein break-up time measurement reproducibility using standard fluorescein strips versus the Dry Eye Test (DET) method. Cornea. 2001;20(8):811-815.

21. Lemp MA. Report of the National Eye Institute/Industry workshop on Clinical Trials in Dry Eyes. CLAO J. 1995;21(4):221-232.

22. Zhao Y, Veerappan A, Yeo S, et al. Clinical Trial of Thermal Pulsation (LipiFlow) in meibomian gland dysfunction with preteatment meibography. Eye Contact Lens. 2016;42(6):339-346.

23. Zhao Y, Xie J, Li J, et al. Evaluation of monocular treatment for meibomian gland dysfunction with an automated thermodynamic system in elderly Chinese patients: a contralateral eye study. $J$ Ophthalmol. 2016;2016:9640643.

24. Greiner JV. A single LipiFlow(R) Thermal Pulsation System treatment improves meibomian gland function and reduces dry eye symptoms for 9 months. Curr Eye Res. 2012;37(4):272-278.

25. Finis D, Hayajneh J, Konig C, Borrelli M, Schrader S, Geerling G. Evaluation of an automated thermodynamic treatment (LipiFlow(R)) system for meibomian gland dysfunction: a prospective, randomized, observer-masked trial. Ocul Surf. 2014;12(2):146-154.

26. Souchier M, Joffre C, Gregoire S, et al. Changes in meibomian fatty acids and clinical signs in patients with meibomian gland dysfunction after minocycline treatment. Br J Ophthalmol. 2008;92(6):819-822.

27. Joffre C, Souchier M, Gregoire S, et al. Differences in meibomian fatty acid composition in patients with meibomian gland dysfunction and aqueous-deficient dry eye. Br J Ophthalmol. 2008;92(1):116-119.

28. Lindsley K, Matsumura S, Hatef E, Akpek EK. Interventions for chronic blepharitis. Cochrane Database Syst Rev. 2012;(5):CD005556.

29. Biller JA, Flores A, Buie T, Mazor S, Katz AJ. Tetracycline-induced esophagitis in adolescent patients. J Pediatr. 1992;120(1):144-145.

30. Carroll LA, Laumann AE. Doxycycline-induced photo-onycholysis. J Drugs Dermatol. 2003;2(6):662-663.

31. Layton AM, Cunliffe WJ. Phototoxic eruptions due to doxycycline - a dose-related phenomenon. Clin Exp Dermatol. 1993;18(5):425-427.

32. Segelnick SL, Weinberg MA. Recognizing doxycycline-induced esophageal ulcers in dental practice: a case report and review. J Am Dent Assoc. 2008;139(5):581-585.

33. Shapiro LE, Knowles SR, Shear NH. Comparative safety of tetracycline, minocycline, and doxycycline. Arch Dermatol. 1997;133(10): 1224-1230.

34. Sloan B, Scheinfeld N. The use and safety of doxycycline hyclate and other second-generation tetracyclines. Expert Opin Drug Saf. 2008;7(5):571-577.

35. Valentin S, Morales A, Sanchez JL, Rivera A. Safety and efficacy of doxycycline in the treatment of rosacea. Clin Cosmet Investig Dermatol. 2009;2:129-140. 


\section{Publish your work in this journal}

Clinical Ophthalmology is an international, peer-reviewed journal covering all subspecialties within ophthalmology. Key topics include: Optometry; Visual science; Pharmacology and drug therapy in eye diseases; Basic Sciences; Primary and Secondary eye care; Patient Safety and Quality of Care Improvements. This journal is indexed on

Submit your manuscript here: http://www.dovepress.com/clinical-ophthalmology-journal
PubMed Central and CAS, and is the official journal of The Society of Clinical Ophthalmology (SCO). The manuscript management system is completely online and includes a very quick and fair peer-review system, which is all easy to use. Visit http://www.dovepress.com/ testimonials.php to read real quotes from published authors. 